\title{
Extraction solvent's effect on biogas production from mixtures of date seed and wastewater sludge
}

\author{
Wameed Radeef ${ }^{1,}{ }^{*}$, Abdallah Shanableh ${ }^{1,2}$, Mohamed Semreen ${ }^{3}$, and Ismail Saadoun ${ }^{4}$ \\ ${ }^{1}$ Department of Civil \& Environmental Engineering, University of Sharjah, 27272 Sharjah, United \\ Arab Emirates \\ ${ }^{2}$ Research Institute of Sciences and Engineering, University of Sharjah, 27272 Sharjah, United Arab \\ Emirates \\ ${ }^{3}$ College of Pharmacy, University of Sharjah, 27272 Sharjah, United Arab Emirates \\ ${ }^{4}$ Department of Applied Biology, University of Sharjah, 27272 Sharjah, United Arab Emirates
}

\begin{abstract}
The objective of this study was to evaluate the impact of extraction solvent on biogas production from mixtures of date (Phoenix dactylifera) seed and wastewater treatment sludge. Date palm seed is a locally produced waste which is used in different useful purposes. Date seed is rich in proteins, carbohydrates and lipids and have the potential for biogas enhancement. In this study, three extraction solvents, hexane, ethyl acetate and Ethanol:water (1:1), were used on two types of date seeds (locally known as Khalas and Khudari). Date seed powder of size 0.425 $0.6 \mathrm{~mm}$ after extraction was mixed with wastewater treatment sludge at dry date seed/dry sludge solids ratios of $0 \%, 10 \%, 20 \%, 40 \%, 60 \%$, and $80 \%$. The study showed a direct relationship between biogas production and ratio of date seeds/wastewater sludge in the mixture. Furthermore, the results confirmed that the quantity of biogas produced from samples containing raw date seed was almost same as the quantity produced from samples containing date seed after extraction. The results revealed that the used extraction solvents had no effect on biogas production
\end{abstract}

\section{Introduction}

Biogas produced from anaerobic digestion (AD) is a promising source of renewable energy that can address the global energy needs [1]. Over the last decades, bio-fuel production from waste products has been a subject of intensive research efforts. Global warming impact and fuel availability have risen many concerns over the use of fossil fuel [2]. Many researchers suggest that bio-fuel production from waste products is a good option for energy recovery [3]. Although there are some limitation in producing bio-fuel from organic wastes, researchers recommend that it is the right time to develop suitable renewable energy sources.

\footnotetext{
*Corresponding author: wradeef@sharjah.ac.ae
} 
Biogas is considered as one of the most environmentally friendly and energy-efficient fuel over other types of biofuel [4]. The production of bio-fuel in the form of methane biogas requires readily available and biodegradable organic feedstocks [5-8]. There are generally two sources of organic matter that are suitable for bio-fuel production, which are: energy crops; and organic waste products.

In this study, wastewater treatment sludge and date palm seeds were co-digested to produce biogas. Both raw and after extraction date seeds were prepared and then codigested with wastewater sludge at different dry weight ratios. Date palm, scientifically called Phoenix dactylifera, seeds are rich in biodegradable organic matter, including Protein (5-5.5\%), Carbohydrates (81-83\%) and Lipids (10-12\%) [9]. Date palm seeds provide a good source of organic matter to the microorganisms involved in $\mathrm{AD}$ process and eventually can potentially contribute to and enhance biogas production from wastewater sludge.

The main focus of this paper is to study the effect of extraction solvents, used on date seeds, on biogas production from mixtures of wastewater sludge and date seeds.

\section{Materials and methods}

Wastewater primary sludge was brought from Dubai Wastewater Treatment Plant. The sludge was obtained from the bottom of the primary sedimentation tank. The total solids (TS) of the sludge was modified to approximately $2 \%$. Then, the sludge was distributed in $750 \mathrm{ml}$ plastic containers and stored in freezer to be used in the future as necessary.

Khalas and khudari date palm seeds were used for biogas production. The date seeds were isolated and thoroughly washed in water to remove date flesh remains. Then, they were dried in the oven at 40 degree for 24 hours. The size of the date seed was adjusted to $0.425-0.6 \mathrm{~mm}$ by using a centrifugal mill with a rotational speed of $10,000 \mathrm{rpm}$ as shown in Figure 1.

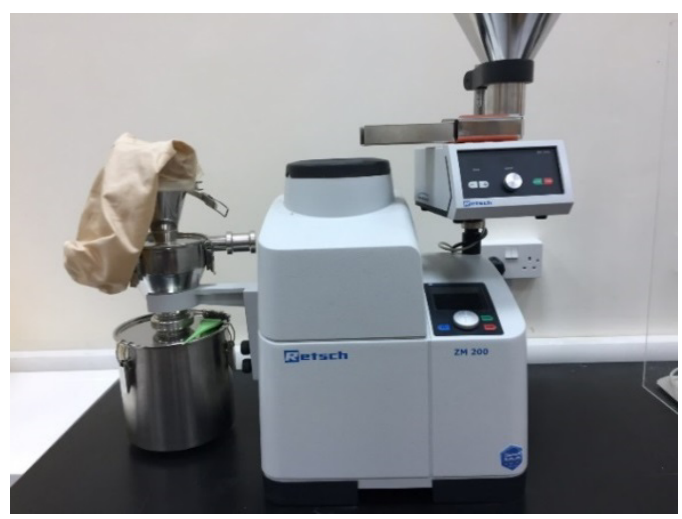

Fig. 1. Ultra Centrifugal. Mill ZM 200

Three extraction solvents, hexane, ethyl acetate and ethanol:water (1:1), were used on two types of date seeds khalas and khudari. Samples of sludge and date palm seeds mixed at different date seed/wastewater total solids dry weight ratios $(0 \%, 10 \%, 20 \%, 40 \%, 60 \%$, and $80 \%$ ) were prepared and filled in $50 \mathrm{ml}$ serum bottles. Then, the serum bottles were capped by using rubber stoppers and aluminium sealing rings and were incubated under mesophilic temperature $\left(35^{\circ} \mathrm{C}\right)$ as shown in Figure 2. 


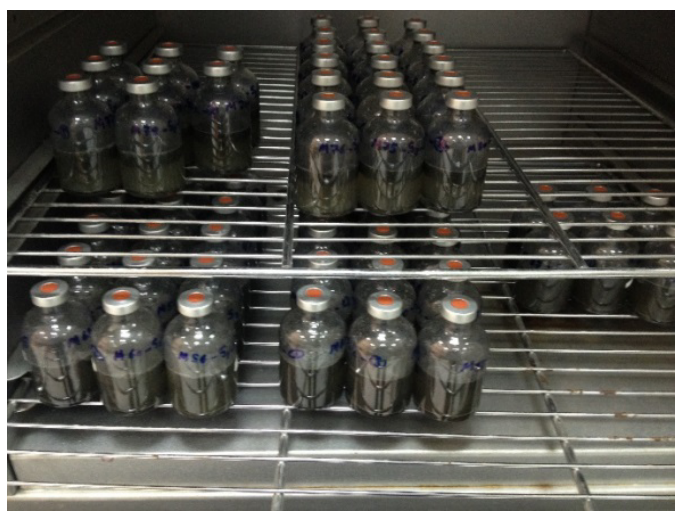

Fig. 2. Samples of Date Seed Co-digested with Wasterwater Treatment Sludge.

Four groups of samples were prepared for each type of the two mentioned types of date seeds (khalas and khudari). The four groups of samples are: wastewater sludge and raw date seed, wastewater sludge and date seed after extraction by hexane, wastewater sludge and date seed after extraction by ethyl acetate, wastewater sludge and date seed after extraction by thanol:water (1:1). All samples were co-digested in duplicates to ensure accuracy.

\section{Results and discussion}

The cumulative biogas production from raw khalas date seed/sludge mixtures after 9 weeks of incubation is shown in Figure 3. The maximum biogas production was achieved from the date seed/sludge samples containing $80 \%$ date seed and the minimum biogas production was from samples containing sludge alone. The volume of the produced biogas increased as the quantity of the added date seed increased. The order of biogas production in terms of date seed ratio in the seed/sludge mixtures was as follows: $80 \%>60 \%>40 \%>20 \%>10 \%>0 \%$.

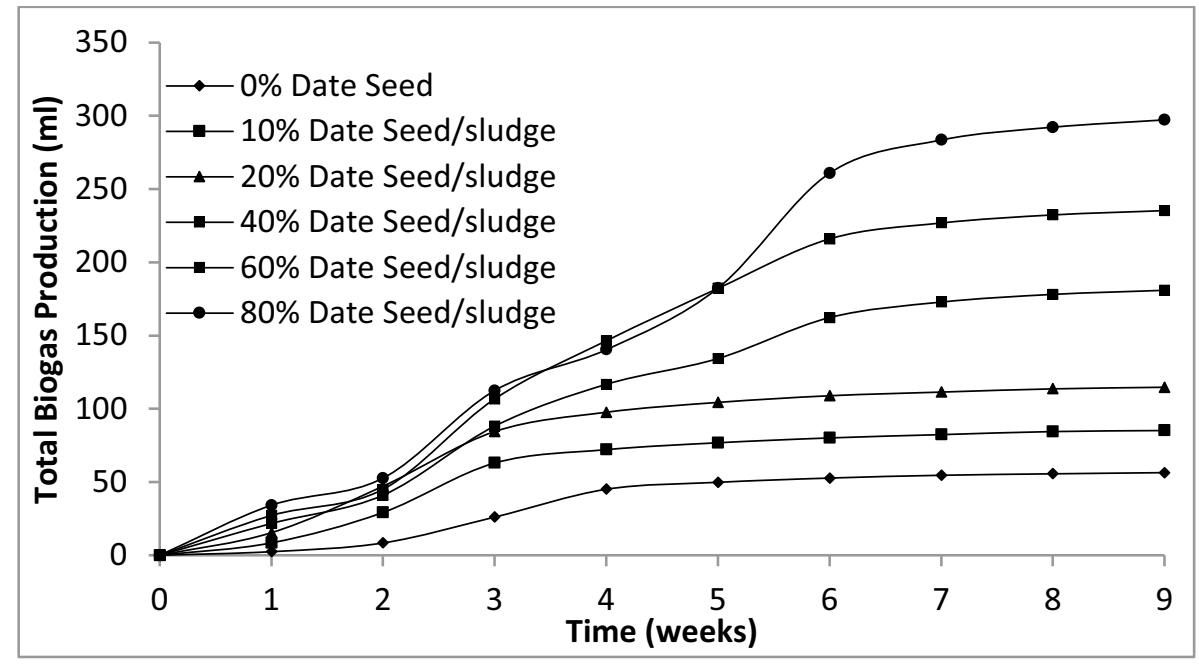

Fig. 3. Cumulative Biogas Production from Mixtures of Raw Khalas Date Seed and Wastewater Sludge.

Samples containing $80 \%$ khalas date seed produced about $527 \%$ biogas compared with $100 \%$ produced by the samples containing $0 \%$ date seed after 9 weeks of incubation. The 
results indicate big enhancement in biogas production. Likewise, the samples containing $80 \%$ khudari date seed produced $539 \%$ biogas compared with $100 \%$ produced by the sludge alone as shown in Figure 4. Both date seed types, khalas and khudari, did almost the same enhancement to the produced biogas when they were added to the wastewater sludge samples.

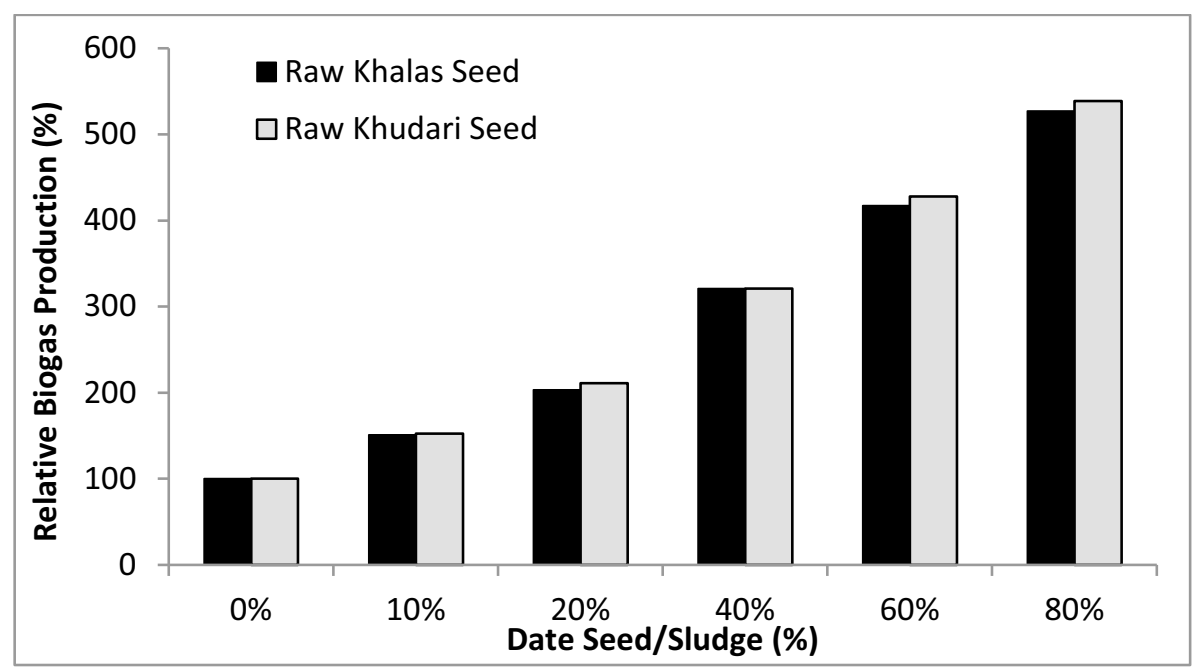

Fig. 4. Comparison of Relative Biogas Production from Mixtures Containing Raw Khalas and Raw Khudari Date Seeds.

The data in Figures 5 and 6 show that raw date seed and date seed after extraction by different solvents had almost the same effect on biogas production. However, raw date seed produced a little bit more biogas than extracted date seed. The three used solvents, hexane, ethyl acetate and ethanol:water (1:1), had no effect on biogas production.

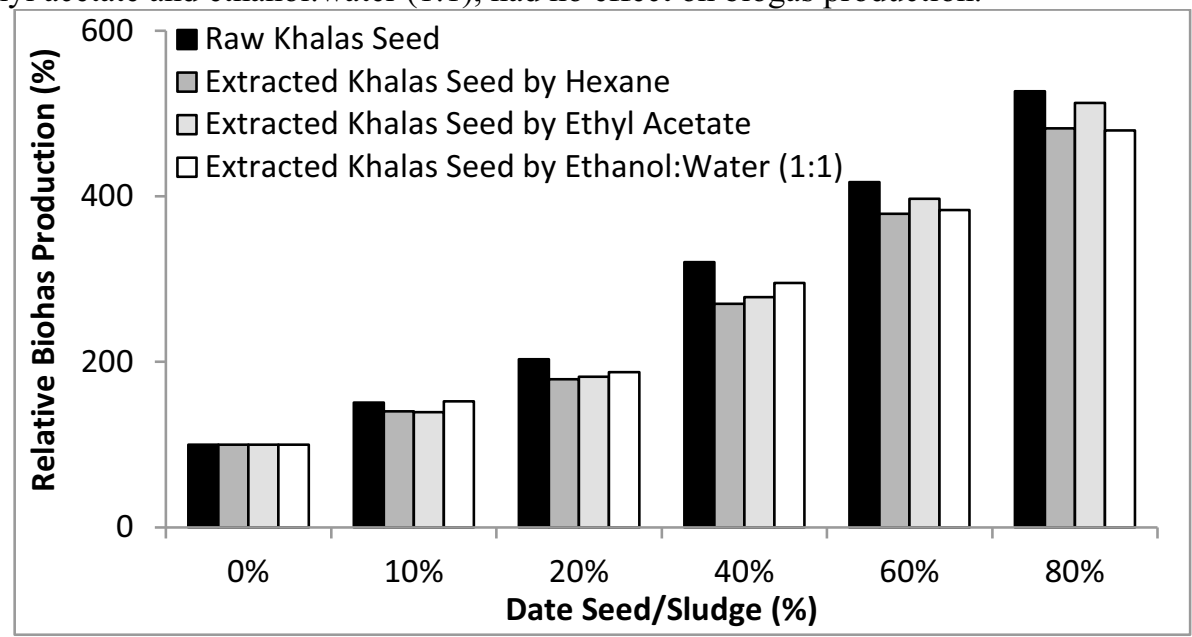

Fig. 5. Comparison of Relative Biogas Production from Mixtures Containing Raw and Extracted Khalas Date Seeds. 


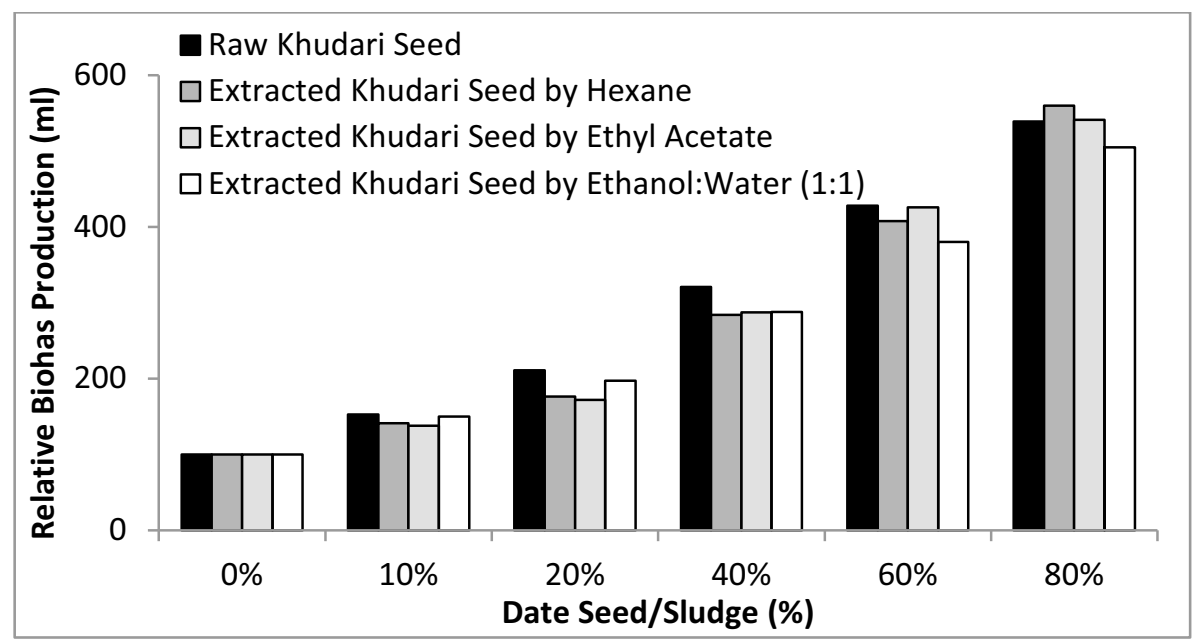

Fig. 6. Comparison of Relative Biogas Production from Mixtures Containing Raw and Extracted Khudari Date Seeds.

\section{Conclusions}

Both date seed types, khalas and khudari, are rich in organic nutrients and had almost the same effect on biogas production. The production of biogas increased as the ratio of date seeds in the mixture increased. The maximum biogas production after 9 weeks of incubation was produced from the date seed/sludge samples containing $80 \%$ date seed and the order of biogas production, in terms of date seed ratios, was $80 \%>60 \%>40 \%>20 \%$ $>10 \%>0 \%$. Samples containing $80 \%$ khalas date seeds produced $527 \%$ biogas and the samples containing $80 \%$ khudari date seeds produced $539 \%$ biogas compared with $100 \%$ produced by the samples containing $0 \%$ date seed. Solvents used in the date seed extraction process had no effect on biogas production.

This study was conducted with support from the Research Institute for Sciences and Engineering (RISE), University of Sharjah, UAE (Project number: 15020401002-P).

\section{References}

1. A. Cuéllar, M. Webber, "Cow power: the energy and emissions benefits of converting manure to biogas", Environ Res Lett 3, 034002 (2008)

2. A.C., Hansen, Q. Zhang, P.W.L. Lyne, Ethanol-diesel fuel blends - a review, Bioresour. Technol 96, 277-285 (2005)

3. Y. Kalogo, and H. Monteith, "Energy and resource recovery from sludge", Global Water Research Coalition, 2008

4. H. Fehrenbach, J. Giegrich, G. Reinhardt, U. Sayer, M. Gretz, K. Lanje, J. Schmitz, "Kriterien einer nachhaltigen Bioenergienutzung im globalen Maßstab", UBAForschungsbericht 206, 41-112 (2008)

5. A.I. Al-Turki, Y.M. El-Hadidi, and S.A. Al-Yahya, "Utilization of date pits as a potential source of biogas and organic fertilizer", Food, Agriculture and Environment 2, 369-374 (2004)

6. G.D. Zupančič, and V. Grilc, "Anaerobic treatment and biogas production from organic waste", Institute for Environmental Protection and Sensors, Slovenia 
7. O.L. Momoh, and L. I. Nwaogazie, "Effect of waste paper on biogas production from co-digestion of cow dung and water hyacinth in batch reactors", J. Appl. Sci. Environ. Manage 12, 95-98 (2008)

8. T.D. Yavini, U.H. Taura, N. Mohammed, and J.M. Namo, "Comparative study of mesophilic biogas production potentials of selected agro-wastes", Int. J. of Eng. and Sci. 3, 1-6 (2014)

9. S. Besbes, C. Blecker, C. Deroanne, G. Lognay, N.E. Drira, N.E., and H. Attia, "Quality characteristics and oxidative stability of date seed oil during storage", Food Sci. Tech. 10, 333-338 (2004) 